\title{
VEGETARIANISMO: UNA CARACTERIZACIÓN ANTROPOMÉTRICA, DIETÉTICA Y MOTIVACIONAL EN ADULTOS VENEZOLANOS
}

\author{
VEGETARIANISM: AN ANTHROPOMETRIC, DIETARY AND MOTIVATIONAL CHARACTERIZATION IN \\ VENEZUELAN ADULTS.
}

\author{
Ekmeiro-Salvador Jesús Enrique*, Arévalo-Vera Cruz Rafael*. \\ *Universidad de Oriente. Puerto La Cruz - Venezuela.
}

\section{RESUMEN}

Introducción: El vegetarianismo es un modelo alimentario en plena expansión, por lo que su estudio reviste interés. Objetivo: caracterizar una muestra de personas que se autodefinen como vegetarianas, de acuerdo a parámetros antropométricos y dietéticos, así como evaluar las razones que motivan su conducta alimentaria. Material y Método: El estado antropométriconutricional se evaluó a través del índice de masa corporal y el diagnóstico de riesgo cardiometabólico. La evaluación dietética se basó en recordatorios de 24 horas y a través de una entrevista personal fueron evaluadas las motivaciones prácticas. Resultados: el $73,21 \%$ de la muestra obtuvo un diagnostico antropométrico normal, y el $18,30 \%$ presenta riesgo cardiometabólico. Según su esquema alimentario se diferenciaron tres grupos: veganos, vegetarianos y flexitarianos; el grupo de vegetarianos fue el único en mostrar un perfil calórico proporcionalmente ajustado a los valores de referencia de energía y nutrientes establecidos para la población venezolana. La deficiencia de vitamina B12 se describe como el principal problema para todos los grupos. Los beneficios sobre la salud, la ecología y el maltrato animal aparecen como razones principales de su orientación alimentaria. Conclusiones: Se evidenció una amplia adecuación de parámetros antropométricos y dietéticos para la población estudiada, pero particularmente el grupo de vegetarianos logró un perfil calórico proporcionado dentro de los valores de referencia establecidos para la población venezolana.

Palabras Clave: Vegano, vegetariano, flexitariano, dieta, nutrición.

\section{ABSTRACT}

Introduction: Vegetarianism is an expanding food model, which is why its study is of interest. Objective: To characterize a sample of people who define themselves as vegetarians, according to anthropometric and dietary parameters, as well as to evaluate the reasons that motivate their eating behavior. Material and method: the anthropometric-nutritional status was evaluated through the body mass index and the diagnosis of cardio metabolic risk. The dietary evaluation was based on $24-$ hour reminders and the practical motivations were evaluated through a personal interview. Results: $73.21 \%$ of the sample obtained a normal anthropometric diagnosis, and $18.30 \%$ presented cardio metabolic risk. According to their diet, three groups were differentiated: vegans, vegetarians and flexitarians; the group of vegetarians was the only one to show a caloric profile proportionally adjusted to the energy and nutrient reference values established for the Venezuelan population. Vitamin B12 deficiency is described as the main problem for all groups. The benefits on health, ecology and animal abuse appear as the main reasons for its food orientation. Conclusion: a wide adaptation of anthropometric and dietary parameters was evidenced for the population studied, but particularly the group of vegetarians achieved a caloric profile provided within the reference values established for the Venezuelan population.

Key words: Vegan, vegetarian, flexitarian, diet, nutrition.

Correspondencia: Jesús Enrique Ekmeiro-Salvador.nutridietsalvador@gmail.com

Recibido: 06 de marzo 2021, aceptado: 28 de julio 2021

(C) Autor2021

(c) (i)

DOI: https://doi.org/10.29105/respyn20.4-6

Citation: Ekmeiro-Salvador J.E., Arévalo-Vera C.R. (2021) Vegetarianismo: una caracterización antropométrica, dietética y motivacional en adultos venezolanos. Revista Salud Pública y Nutrición, 20 (4), 57-72. 


\section{Artículo Original}

\section{Introducción}

El crecimiento del vegetarianismo, como modelo alimentario y como estilo de vida, forma parte de una tendencia global ampliamente descrita a través de una vasta literatura académica disponible. Sus prácticas constituyen parte del patrimonio culinariocultural en muchos países del Sur Global, mientras que en los del Norte Global representan una alternativa de cambio generalmente ligada a la filosofía de la vida, preocupación por la degradación del medio ambiente, compasión por los animales, cuidados de la salud o motivos religiosos (Miranda, Gomes, Morais, Tonetti y Vassimon, 2013).

El vegetarianismo cubre amplias prácticas alimentarias y de vida. Se caracteriza por su apego a criterios espirituales, filosóficos, morales y emocionales (Orellana, Sepúlveda y Denegri, 2013; González Saura, 2018) que proponen otras formas de ver el mundo y organizar la manera de alimentarse. En gran medida puede ser considerado parte de un estilo de vida o de identidad (Brignardello, Heredia, Ocharán y Durán, 2013; Parker y Vadiveloo, 2019) e incluso, en el caso del veganismo, se asume como postura política traducida en un activismo que ha comenzado a influir sobre el derecho y las leyes ciudadanas; así como ha generado un nuevo tipo de gastronomía en auge (Labbé Yáñez, 2016) asociada a una visión más ecológica de los sistemas económicos y el consumo. Los activistas veganos han sido objeto de estigma, estereotipos y actitudes negativas, al manifestar y defender creencias éticas importantes (Povey, Wellens y Conner, 2001; Cole y Morgan, 2011; Bresnahan, Zhuang y Zhu, 2016) pero que con el tiempo han sido finalmente respaldadas y ampliamente reconocidas por la ciencia.

Frecuentemente estos estilos de vida también tienen relación con motivos religiosos; históricamente hinduistas y hebreos proponen a sus fieles prácticas vegetarianas para alcanzar o mantener la purificación, así como los Adventistas del Séptimo Día fundamentan su propuesta de vida equilibrada, activa y longeva haciendo especial mención a la alimentación como una columna base para obtener la salud integral que ellos sugieren y practican (Guzmán Cáceres, 2017)

La popularidad del vegetarianismo está creciendo especialmente entre mujeres, adolescentes y adultos jóvenes (Le Roy y Díaz San Martín, 2010;
Brignardello, et al., 2013; Bravo, Ibarra y Paredes, 2014; Parker y Vadiveloo,2019), poblaciones urbanas y con fuerte capital cultural, como ejecutivos o de alto nivel educativo (Centre de recherche pour l'étude et l'observation des conditions de vie [CRÉDOC], 2019); debido particularmente a la divulgación cada vez más frecuente, en medios y redes de comunicación social, de estudios que evalúan el impacto sobre el medio ambiente y sobre los recursos hídricos de la producción intensiva de alimentos de origen animal (Pilis, Stec, Zych y Pilis, 2014), la ineficiencia de la producción de carne y su vínculo con el calentamiento global (Bravo, et al., 2014; Sánchez Mendoza, Flores Villalba, Rodríguez Hernández, Anaya Escalera y Contreras 2020).

Las dietas a base de plantas son más sostenibles desde el punto de vista medioambiental que las dietas ricas en productos animales porque utilizan menos recursos naturales y generan mucho menos daño (Melina, Craig y Levin, 2016); por tanto son una forma de reducir la huella ecológica, dado que estas dietas producen emisiones en cantidad bastante menor de gases de efecto invernadero en comparación a las dietas omnívoras (Rosi, et al., 2017; Chai, Van der Voort, Grofelnik, Eliasdottir, Klöss y Pérez-Cueto, 2019).

Las modalidades del vegetarianismo son en su mayoría producto de creencias éticas sólidas sobre el bienestar animal, referidas a la matanza y el uso excesivo o innecesario de los animales para alimentarnos y vestirnos. Igualmente se ha incrementado la conciencia pública sobre el manejo industrial de los animales, donde frecuentemente están bajo condiciones de suciedad, aglomerados en lugares donde no pueden moverse o llevar a cabo sus comportamientos naturales, sufriendo problemas de salud o llegando a morir debido a que son criados para producir leche o huevos a un rango mayor al que sus cuerpos pueden responder naturalmente. Elegir una dieta vegana es una demostración diaria de compasión por los animales de todo el planeta (Calderón Aravena 2017; Hölker, Von Meyer-Höfer y Spiller, 2019); se estima que actualmente alrededor del $46 \%$ de los vegetarianos en Estados Unidos son veganos (Clifford y Kozil, 2017).

La epidemiología nutricional viene trabajando con todos estos conceptos, demostrando la significativa conexión entre los estilos de vida que promueven 
dietas vegetarianas y su impacto positivo sobre la salud (Le, Sabaté, Singh y Jaceldo-Siegl, 2018). Las dietas bajas en carnes y derivados, pero especialmente las dietas vegetarianas que los excluyen por completo, están asociadas al aumento de la esperanza de vida y pueden influir en los niveles circulantes de biomarcadores inflamatorios, reduciendo así el riesgo de enfermedades crónicas no transmisibles al alcanzar mejores niveles de tensión arterial, perfil lipídico y glucosa en sangre; por lo tanto, menor predisposición a padecer diabetes mellitus tipo 2, enfermedades cardiovasculares, obesidad, hipertensión, hiperlipidemia y cáncer. Además, existen beneficios sobre enfermedades autoinmunes como la artritis reumatoide. Mientras que en el caso contrario y con reducida ingesta de alimentos de origen vegetal, aumentan considerablemente todos estos riesgos (Kahleova, Levin y Barnard, 2017; Rojas Allende, Figueras Díaz, y Durán Agüero, 2017; Dinu, Abbate, Gensini, Casini, y Sofi, 2017; Menzel, et al., 2020). De igual modo, como en cualquier otra dieta o patrón alimentario propuesto, si no se dispone una adecuada formulación técnica sus seguidores podrían exponerse a riesgos importantes en cuanto a la biodisponibilidad adecuada de algunos nutrientes, en este caso de aminoácidos esenciales, ácidos grasos poliinsaturados (AGP) omega $3(\mathrm{n}-3)$ y determinados micronutrientes tales como hierro, zinc y selenio, o déficit de vitaminas B12 y D. Esto se asocia a riesgo de déficit nutricional, por lo que se hace necesaria una adecuada supervisión profesional y educación nutricional continua (Pilis, et al., 2014; Dinu, et al., 2017; Sebastiani, et al., 2019; Martínez Biarge, 2019).

En todo el mundo la prevalencia de vegetarianos varía considerablemente, y aunque generalmente representan un porcentaje discreto de la población de cada país, es un sector consolidado en franca expansión. En Europa, donde el movimiento es muy activo, llegan a representar hasta el 5,6\% del promedio ponderado en cuatro de los países más poblados, en Reino Unido 8,0\%, Alemania 5,6\%, Francia 5,2\% y en España 2,8\%. Adicionalmente, una proporción aún mayor de personas muestra interés en integrarse al vegetarianismo al definirse como flexitarianos; en Alemania alcanzan el 26,0\%, Francia 20\%, España $23 \%$ y en Reino Unido hasta un $19 \%$ de la población estudiada (CRÉDOC, 2019). En India, el país más representativo, hasta el $40 \%$ de las personas son vegetarianas, el 3,3 \% en Estados Unidos y hasta el 14\% de la población brasileña está calificada como tal (Oregon State University, 2011; Ruby, 2012; Hrynowski, 2019; Hargreaves, Araújo, Nakano y Zandonadi, 2020).

Aunque en Latinoamérica existen pocos estudios recientes que estimen el porcentaje de vegetarianos, algunos datos de consultoras de mercadeo sugieren que podría tratarse de hasta un $8 \%$ de la población general, siendo México el país con más vegetarianos de Latinoamérica con $19 \%$, de los cuales un $9 \%$ se consideran veganos (The Nielsen Company, 2016).

En Venezuela, la falta de profesionales especializados en el área, además de la escasez de estudios sobre el vegetarianismo, nos lleva a considerar muy importante la producción de información sobre el tema. Así, el presente estudio tuvo como objetivo identificar en participantes del área metropolitana de Puerto La Cruz los diferentes tipos de prácticas vegetarianas, analizando la calidad nutritiva de sus dietas, el impacto sobre el estado nutricional, así como las motivaciones que les animan a seguir este tipo de esquema alimentario.

\section{Material y Método}

Se trata de un estudio descriptivo, transversal y con abordaje cuantitativo; que cuenta con la aprobación del Comité de Bioética e Investigación y donde todos los participantes firmaron el término de libre consentimiento. La selección de los sujetos se derivó de conferencias y clases magistrales sobre vegetarianismo dictadas en universidades y oficinas gubernamentales, así como en un restaurante vegetariano y en una escuela de gastronomía del Área Metropolitana de Puerto La Cruz, en Venezuela, entre Julio de 2018 y febrero de 2020; en ellas se informaba sobre la naturaleza de nuestro estudio y la necesidad de contar con un voluntariado participante. Los criterios de inclusión de la investigación fueron individuos mayores de 18 años, sanos, de ambos sexos y que se consideraran vegetarianos; mientras que los de exclusión se enfocaron en que esta práctica alimentaria fuese de carácter continuo y que dispusieran de tiempo suficiente para asistir a las entrevistas para cumplimentar la información requerida.

Las personas participantes fueron convocadas individualmente para una evaluación presencial, que 
incluyó una entrevista que permitía conocer las principales razones por las cuales se sentía motivadas por las prácticas alimentarias vegetarianas, cómo las desarrollaban y el tiempo que llevaban de manera ininterrumpida dentro de la misma. Para realizar la antropometría, se tomaron medidas de masa corporal (kg) empleando la Báscula portátil Tanita ${ }^{\circledR}$, capacidad $200 \mathrm{~kg}$, así como la altura (m) obtenida con estadiómetro portátil digital prototipo de la Universidad de Córdoba- España. Con estos datos se calculó el índice de masa corporal (IMC) en kg / m2, para la clasificación del estado nutricional según la Organización Mundial de la Salud. Igualmente se midió el perímetro de cintura, para el diagnóstico de riesgo cardiometabólico en adultos, tomando como patrón la clasificación de la Federación Internacional de Diabetes.

Para la evaluación de la ingesta de nutrientes se recabaron dos recordatorios de 24 horas en días no consecutivos de una misma semana a cada participante; en ellos quedaron reportados todos los alimentos que fueron consumidos durante el día anterior, describiendo si habían sido alimentos individuales y crudos o preparados y bebidas, naturales o con algún nivel de proceso industrial, si los habían consumido dentro o fuera del hogar, detalles sobre su preparación o receta, así como la cantidad consumida. Se solicitó además información específica sobre el consumo de suplementos multivitamínicos.

Toda la información fue recogida en un cuestionario abierto o predeterminado (en formato de papel o digital) a través de una entrevista presencial manejada por nutricionistas dietistas previamente capacitadas y siguiendo la metodología de pasos múltiples (United States Department of Agriculture, 2014). Para precisar las cantidades consumidas, se apoyaron en alimentos modelados y figuras geométricas que cuentan con el peso estandarizado del alimento que representan; además, se emplearon un álbum de fotografías de utensilios caseros en tamaño real y algunas unidades de ayuda como tazas, vasos y cucharas. Las nutricionistas transformaron estas medidas caseras a gramos y mililitros utilizando tablas de medidas y raciones venezolanas (Escuela de Nutrición y Dietética UCV, 2002), para luego hacer una revisión final cruzada de estas conversiones con otro colega.
Estadísticamente el consumo de cada participante fue analizado individualmente realizando un estudio de frecuencia para cada uno de los alimentos reportados en ambas visitas, determinando la moda estadística, que arrojó el valor de gramaje más representativo para cada alimento consumido. La posterior información nutricional de estos datos de consumo de alimentos se obtuvo a través del programa Food Processor® utilizando la Tabla de Composición de Alimentos venezolana (Instituto Nacional de Nutrición [INN], 2015) para la cantidad neta de macronutrientes (energía, proteínas, grasa, carbohidratos) y micronutrientes (calcio, hierro, magnesio, zinc, vitamina A) de cada uno de los participantes del estudio, y a través de la Tabla de Composición de Alimentos de Centroamérica (Instituto de Nutrición de Centro América y Panamá [INCAP], 2012) para obtener específicamente las cantidades netas de AGP, que incluyen omega 3 y omega 6 , y vitamina B12 consumidas.

En esta investigación el análisis del consumo de calorías y nutrientes seleccionados se basó en los valores de referencia del Instituto Nacional de Nutrición de Venezuela (INN, 2012), determinando para cada tipo de dieta estudiada tanto el perfil calórico, que engloba los macronutrientes (energía, proteínas, grasas y carbohidratos), como las fuentes de las proteínas consumidas, animal o vegetal, desagregando la participación de las mismas según los diferentes tipos de dietas estudiadas.

Para evaluar la contribución calórica porcentual de los macronutrientes se consideraron las recomendaciones para la población venezolana: entre $11-14 \%$ para las proteínas, $20-30 \%$ para las grasas y $56-69 \%$ para los carbohidratos; y la adecuación de su consumo a través de tres categorías: adecuado (95-105\%), inadecuado por déficit $(<95 \%)$ e inadecuado por exceso $(>105 \%)$. En el caso de los micronutrientes se establecieron como criterios los de adecuado (85-115\%), inadecuado por déficit $(<85 \%)$ e inadecuado por exceso $(>115 \%)$ (Rojas, 2009).

Se utilizó estadística inferencial para determinar diferencias en las variables, considerando como un $\mathrm{p}$ significativo aquel $<0,05$ haciendo uso del Chi cuadrado; así como el paquete estadístico SPSS versión 20 para analizar toda la información derivada 
de las encuestas; así como tabular y graficar los resultados obtenidos.

\section{Resultados}

Del total de personas evaluadas 84 sujetos fueron del sexo masculino ( $37,5 \%$ ) y 140 del sexo femenino $(62,5 \%)$; de ese total $(n=224)$ de muestra validada el $12,94 \%(\mathrm{n}=29)$ correspondieron a personas veganas, que no consumen ningún tipo de carne ni productos de origen animal, el 33,03\% $(n=74)$ fueron definidos como vegetarianos propiamente dichos, cuyas dietas excluyen carnes pero no aquellos productos de origen animal (huevos, leche, miel) que no impliquen el sacrificio animal, y finalmente un $54,01 \%(\mathrm{n}=121)$ correspondieron a población flexitariana, que basan su dieta mayoritariamente en productos de origen vegetal pero que pueden incluir algunos productos de origen animal y eventualmente pequeñas cantidades de carnes, especialmente de animales marinos.

El diagnóstico del estado nutricional (ver Tabla 1) arrojo que el $73,21 \%$ de la muestra presenta un diagnóstico normal $(n=164)$, el $16,07 \%$ presenta sobrepeso $(n=36)$ principalmente a expensas del grupo de flexitarianos, al igual que el 8,02\% de la muestra cursa con algún tipo de obesidad $(n=18)$. El $2,67 \%$ de las personas evaluadas fueron diagnosticadas con déficit $(n=6)$ principalmente a expensas de los grupos de vegetarianos y veganos. El $18,30 \%$ de los participantes presentaron riesgo cardiometabólico $(n=41)$, concentrando el grupo de flexitarianos el 55,28\% ( $\mathrm{n}=29)$ de los diagnosticados; los vegetarianos reúnen $33,69 \%(n=11)$ de los casos mientras que los veganos resultaron el $8,33 \%(n=1)$.

Tabla 1: Estado nutricional según tipo de dieta. Puerto La Cruz, 2021

\begin{tabular}{|c|c|c|c|c|c|c|}
\hline \multirow{2}{*}{ Tipo Dieta } & \multicolumn{5}{|c|}{ Diagnóstico Antropométrico } & \multirow{2}{*}{$\begin{array}{l}\text { Riesgo Cardio } \\
\text { metabólico }\end{array}$} \\
\hline & Déficit & Normal & Sobrepeso & Obesidad I & Obesidad II & \\
\hline \multirow{2}{*}{$\begin{array}{c}\text { Veganos } \\
M=12 \\
F=17\end{array}$} & $\begin{array}{r}1 \\
(8,33 \%)\end{array}$ & $\begin{array}{r}10 \\
(83,33 \%)\end{array}$ & $\begin{array}{r}1 \\
(8,33 \%)\end{array}$ & $\begin{array}{r}0 \\
(0,00 \%)\end{array}$ & $\begin{array}{r}0 \\
(0,00 \%)\end{array}$ & $\begin{array}{r}1 \\
(8,33 \%)\end{array}$ \\
\hline & $\begin{array}{r}2 \\
(11,76 \%)\end{array}$ & $\begin{array}{r}14 \\
(82,35 \%)\end{array}$ & $\begin{array}{r}1 \\
(5,88 \%)\end{array}$ & $\begin{array}{r}0 \\
(0,00 \%)\end{array}$ & $\begin{array}{r}0 \\
(0,00 \%)\end{array}$ & $\begin{array}{r}0 \\
(0,00 \%)\end{array}$ \\
\hline \multirow{2}{*}{$\begin{array}{c}\text { Vegetarianos } \\
\qquad \begin{array}{l}M=28 \\
F=46\end{array}\end{array}$} & $\begin{array}{r}1 \\
(3,57 \%)\end{array}$ & $\begin{array}{r}20 \\
(71,42 \%)\end{array}$ & $\begin{array}{r}5 \\
(17,85 \%)\end{array}$ & $\begin{array}{r}1 \\
(3,57 \%)\end{array}$ & $\begin{array}{r}1 \\
(3,57 \%)\end{array}$ & $\begin{array}{r}7 \\
(25,00 \%)\end{array}$ \\
\hline & $\begin{array}{r}2 \\
(4,34 \%)\end{array}$ & $\begin{array}{r}37 \\
(78,26 \%)\end{array}$ & $\begin{array}{r}4 \\
(8,69 \%)\end{array}$ & $\begin{array}{r}2 \\
(4,34 \%)\end{array}$ & $\begin{array}{r}1 \\
(2,17 \%)\end{array}$ & $\begin{array}{r}4 \\
(8,69 \%)\end{array}$ \\
\hline \multirow{2}{*}{$\begin{array}{c}\text { Flexitarianos } \\
\qquad \begin{array}{l}M=44 \\
F=77\end{array}\end{array}$} & $\begin{array}{r}0 \\
(0,00 \%)\end{array}$ & $\begin{array}{r}26 \\
(59,09 \%)\end{array}$ & $\begin{array}{r}11 \\
(25,00 \%)\end{array}$ & $\begin{array}{r}5 \\
(11,36 \%)\end{array}$ & $\begin{array}{r}2 \\
(4,54 \%)\end{array}$ & $\begin{array}{r}18 \\
(40,90 \%)\end{array}$ \\
\hline & $\begin{array}{r}0 \\
(0,00 \%)\end{array}$ & $\begin{array}{r}57 \\
(74,02 \%)\end{array}$ & $\begin{array}{r}14 \\
(18,18 \%)\end{array}$ & $\begin{array}{r}4 \\
(5,19 \%)\end{array}$ & $\begin{array}{r}2 \\
(2,59 \%)\end{array}$ & $\begin{array}{r}11 \\
(14,28 \%)\end{array}$ \\
\hline $\begin{array}{c}\text { Total } \\
224\end{array}$ & $\begin{array}{r}6 \\
(2,67 \%)\end{array}$ & $\begin{array}{r}164 \\
(73,21 \%)\end{array}$ & $\begin{array}{r}36 \\
(16,07 \%)\end{array}$ & $\begin{array}{r}12 \\
(5,35 \%)\end{array}$ & $\begin{array}{r}6 \\
(2,67 \%)\end{array}$ & $\begin{array}{r}41 \\
(18,30 \%)\end{array}$ \\
\hline
\end{tabular}

Fuente: encuesta
El aporte promedio de energía suministrada por los macronutrientes fue de $1877 \mathrm{kcal} /$ persona/día en el grupo de veganos, $2094 \mathrm{kcal} /$ persona/día para los vegetarianos y $2197 \mathrm{kcal} / \mathrm{persona} /$ día entre los flexitarianos. La fórmula calórica promedio para cada uno de los grupos de participantes según su tipo de dieta se esquematiza en la Figura 1, donde se observa que el grupo de vegetarianos es el único ajustado a las recomendaciones venezolanas de contribución calórica porcentual por macronutrientes. Frente a esta referencia, el grupo de veganos estudiados mostró una dieta deficiente en proteínas y grasas, pero excesiva en hidratos de carbono. Por el contrario, el grupo de flexitarianos reportó una fórmula excesiva en proteínas y grasas, pero deficitaria en carbohidratos.

Al respecto de los macronutrientes la mayoría de muestra estudiada $(80,35 \%)$ presentó un consumo adecuado de energía, con un $17,85 \%$ de la misma descrita en exceso para su requerimiento. En el caso de las proteínas el $70,48 \%$ se ubicó dentro del margen adecuado; la prueba chi2 reportó además diferencia significativa según el tipo de dieta $(\mathrm{p}<$ $0,05)$ ya que los flexitarianos presentaron una mayor inadecuación por exceso para este nutriente. También se reportó adecuación para los consumos de grasas $(83,03 \%)$ y carbohidratos $(80,80 \%)$ en todos los grupos estudiados. Aunque el $84,37 \%$ de los participantes demostraron ingestas adecuadas de AGP, se encontraron diferencias significativas entre los diferentes tipos de dietas $(\mathrm{p}<0,05)$ reportándose déficits marcados para los grupos de vegetarianos y veganos (ver Tabla 2).

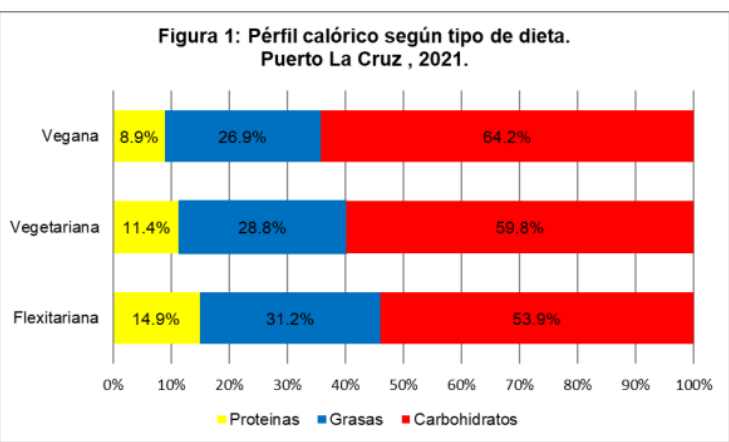

En el grupo de minerales estudiados se observó una amplia adecuación para el calcio $(90,17 \%)$ y el zinc $(89,73 \%)$, y un poco más discreta para magnesio $(74,10 \%)$ y hierro $(72,32 \%)$ en la totalidad de 
personas participantes. Se encontraron diferencias significativas entre los tipos de dietas $(\mathrm{p}<0,05)$ para el déficit de calcio y zinc en veganos; de igual modo significativa para los excesos de hierro y magnesio en los grupos de vegetarianos y veganos (ver Tabla 2).

De las vitaminas consideradas para esta investigación presentaron altos porcentajes de adecuación general tanto la A $(81,69 \%)$ como la C $(88,39 \%)$; mientras que la vitamina B12, aunque alcanza adecuación entre el $69,64 \%$ de la muestra, reporta importantes deficiencias de consumo para todos los tipos de dietas, que se hacen significativas $(p<0,05)$ en el caso de vegetarianos y veganos, quienes resultaron con los mayores déficits (ver Tabla 2).

Cuando los encuestados fueron preguntados sobre la suplementación imprescindible para este tipo de dietas encontramos diferencias importantes entre los tres grupos: el $100 \%$ de los veganos $(n=29)$ respondieron acertadamente, un $32,4 \%$ de vegetarianos $(n=24)$ afirmaron conocer el carácter de la suplementación, y solo un $23,1 \%$ de flexitarianos $(\mathrm{n}=28)$ manifestó estar informado sobre los requisitos adecuados de suplementación. Aunque el $36,1 \%(n=81)$ de la muestra estudiada conocen la importancia de la suplementación, de igual modo un $46,9 \%$ de la misma $(n=38)$ afirmaron no cumplir de manera regular con la misma.

\begin{tabular}{|c|c|c|c|c|c|}
\hline \multirow{2}{*}{\multicolumn{2}{|c|}{ Adecuación de Nutrientes - }} & \multicolumn{3}{|c|}{ Dietas } & \multirow{2}{*}{$\begin{array}{c}\text { Total } \\
\text { General }\end{array}$} \\
\hline & & Veganos* & Vegetarianos** & Flexitarianos*** & \\
\hline \multirow{4}{*}{ Energía } & Déficit & $3,44 \%$ & $2,70 \%$ & $0,82 \%$ & $1,78 \%$ \\
\hline & Adecuado & $93,10 \%$ & $82,43 \%$ & $76,03 \%$ & $80,35 \%$ \\
\hline & Exceso & $3,44 \%$ & $14,86 \%$ & $23,14 \%$ & $17,85 \%$ \\
\hline & Déficit & $6,89 \%$ & $2,70 \%$ & $0,00 \%$ & $1,78 \%$ \\
\hline \multirow[t]{3}{*}{ Proteína } & Adecuado & $93,10 \%$ & $74,32 \%$ & $63,63 \%$ & $70,98 \%$ \\
\hline & Exceso & $0,00 \%$ & $22,97 \%$ & $36,36 \%$ & $27,23 \%$ \\
\hline & Déficit & $0,00 \%$ & $1,35 \%$ & $0,82 \%$ & $0,89 \%$ \\
\hline \multirow[t]{3}{*}{ Grasas } & Adecuado & $100,00 \%$ & $89,18 \%$ & $75,20 \%$ & $83,03 \%$ \\
\hline & Exceso & $0,00 \%$ & $9,45 \%$ & $23,96 \%$ & $16,07 \%$ \\
\hline & Déficit & $10,34 \%$ & $16,21 \%$ & $4,95 \%$ & $9,37 \%$ \\
\hline \multirow[t]{3}{*}{ AGP } & Adecuado & $89,65 \%$ & $78,37 \%$ & $85,12 \%$ & $84,37 \%$ \\
\hline & Exceso & $0,00 \%$ & $5,40 \%$ & $9,91 \%$ & $6,25 \%$ \\
\hline & Déficit & $0,00 \%$ & $4,05 \%$ & $1,65 \%$ & $2,23 \%$ \\
\hline \multirow[t]{3}{*}{ Carbohidratos } & s Adecuado & $100,00 \%$ & $83,78 \%$ & $74,38 \%$ & $80,80 \%$ \\
\hline & Exceso & $0,00 \%$ & $12,16 \%$ & $23,96 \%$ & $16,96 \%$ \\
\hline & Déficit & $13,79 \%$ & $0,00 \%$ & $0,82 \%$ & $2,23 \%$ \\
\hline \multirow[t]{3}{*}{$\mathrm{Ca}$} & Adecuado & $86,20 \%$ & $93,24 \%$ & $89,25 \%$ & $90,17 \%$ \\
\hline & Exceso & $0,00 \%$ & $6,75 \%$ & $9,91 \%$ & $7,58 \%$ \\
\hline & Déficit & $3,44 \%$ & $1,35 \%$ & $0,82 \%$ & $1,33 \%$ \\
\hline \multirow[t]{3}{*}{$\mathrm{Fe}$} & Adecuado & $58,62 \%$ & $68,91 \%$ & $77,68 \%$ & $72,32 \%$ \\
\hline & Exceso & $37,93 \%$ & $29,72 \%$ & $21,48 \%$ & $26,33 \%$ \\
\hline & Déficit & $0,00 \%$ & $0,00 \%$ & $0,00 \%$ & $0,00 \%$ \\
\hline \multirow[t]{3}{*}{ Mg } & Adecuado & $62,06 \%$ & $71,62 \%$ & $78,51 \%$ & $74,10 \%$ \\
\hline & Exceso & $37,93 \%$ & $28,37 \%$ & $21,48 \%$ & $25,89 \%$ \\
\hline & Déficit & $13,79 \%$ & $14,86 \%$ & $5,78 \%$ & $9,82 \%$ \\
\hline \multirow[t]{3}{*}{$\mathrm{Zn}$} & Adecuado & $86,20 \%$ & $85,13 \%$ & $93,38 \%$ & $89,73 \%$ \\
\hline & Exceso & $0,00 \%$ & $0,00 \%$ & $0,82 \%$ & $0,45 \%$ \\
\hline & Déficit & $6,89 \%$ & $9,45 \%$ & $9,91 \%$ & $9,37 \%$ \\
\hline \multirow[t]{3}{*}{ A } & Adecuado & $93,10 \%$ & $78,37 \%$ & $80,99 \%$ & $81,69 \%$ \\
\hline & Exceso & $0,00 \%$ & $12,16 \%$ & $9,09 \%$ & $8,92 \%$ \\
\hline & Déficit & $0,00 \%$ & $1,35 \%$ & $2,47 \%$ & $1,78 \%$ \\
\hline \multirow[t]{3}{*}{ c } & Adecuado & $89,65 \%$ & $93,24 \%$ & $85,12 \%$ & $88,39 \%$ \\
\hline & Exceso & $10,34 \%$ & $5,40 \%$ & $12,39 \%$ & $9,82 \%$ \\
\hline & Déficit & $75,86 \%$ & $33,78 \%$ & $17,35 \%$ & $30,35 \%$ \\
\hline \multirow[t]{2}{*}{ B12 } & Adecuado & $24,13 \%$ & $66,21 \%$ & $82,64 \%$ & $69,64 \%$ \\
\hline & Exceso & $0,00 \%$ & $0,00 \%$ & $0,00 \%$ & $0,00 \%$ \\
\hline
\end{tabular}

El consumo habitual de proteína según su origen resultó bastante diferenciado según el tipo de dieta, así en el grupo de veganos fue de $100 \%$ proteína vegetal, los vegetarianos reportaron un $32,1 \%$ de proteína animal y $67,9 \%$ vegetal, mientras que en los flexitarianos fue significativamente menor la diferencia entre $49,6 \%$ de proteína animal y $50,4 \%$ de origen vegetal; la totalidad de la muestra promedia un consumo de $68,9 \mathrm{~g} /$ proteína/día y solamente en el grupo de hombres flexitarianos se observa un consumo mayor de proteína de origen animal sobre la de origen vegetal (ver Tabla 3 ). 
Tabla 3: Consumos promedios de proteínas según tipo de dieta. Puerto La Cruz, 2021.

\begin{tabular}{|c|c|c|c|}
\hline \multirow{2}{*}{\multicolumn{2}{|c|}{ Dietas }} & Proteína Animal & Proteína Vegetal \\
\hline & & (g) & (g) \\
\hline \multirow{2}{*}{ Vegana* } & Hombres & 0 & $46.3 \pm 12.7$ \\
\hline & Mujeres & 0 & $40,8 \pm 10,9$ \\
\hline \multirow{2}{*}{ Vegetariana** } & Hombres & $19,2 \pm 8,2$ & $42,4 \pm 13,3$ \\
\hline & Mujeres & $17,9 \pm 6,9$ & $36,3 \pm 11,0$ \\
\hline \multirow{2}{*}{ Flexitariana*** } & Hombres & $43,4 \pm 12,6$ & $40,5 \pm 12,6$ \\
\hline & Mujeres & $32,2 \pm 9,4$ & $34,5 \pm 11,9$ \\
\hline Total General & & $28,7 \pm 9,2$ & $40,2 \pm 10,7$ \\
\hline \multicolumn{4}{|l|}{ Fuente: encuesta } \\
\hline \multicolumn{4}{|l|}{${ }^{*} n=29$} \\
\hline \multicolumn{4}{|l|}{$* * n=74$} \\
\hline \multicolumn{4}{|l|}{$* * * n=121$} \\
\hline
\end{tabular}

La adhesión a alguno de los tipos de dieta vegetariana varía de manera significativa según el grupo estudiado. Para el 33,47\% ( $\mathrm{n}=81)$ de los flexitarianos la principal razón es la salud, seguida en un $26,85 \%$ $(n=65)$ por la necesidad de adelgazar y en el $26,44 \%$ $(n=64)$ de los participantes por razones de economía. Para los vegetarianos también la salud con el $33,1 \%(n=49)$ y la economía con $24,32 \%(n=36)$ son las principales razones para seguir este esquema alimentario, más desmarcadas de la necesidad de adelgazar que fue señalada por el $16,21 \%(n=24)$ de los encuestados. La totalidad de la muestra de veganos coincidió al señalar dos razones principales que fundamentan su esquema de alimentación y estilo de vida, y fueron tanto el respeto a los animales como las implicaciones de la alimentación sobre la ecología, alcanzando un $50 \%(n=29)$ cada criterio (ver Figura 2).

Cabe destacar que ningún participante, de ningún grupo, señaló razones religiosas como motivación base para su conducta alimentaria. Sin embargo, el $43,75 \%(n=98)$ del total de las personas estudiadas reportaron pertenecer a grupos religiosos que promueven dentro de sus prácticas y fundamentos enfoques alimentarios de tipo vegetariano: judíos $(\mathrm{n}=7)$, hinduismo krishna $(\mathrm{n}=11)$, taoístas $(\mathrm{n}=19)$ y adventistas del 7 mo día $(\mathrm{n}=61)$.

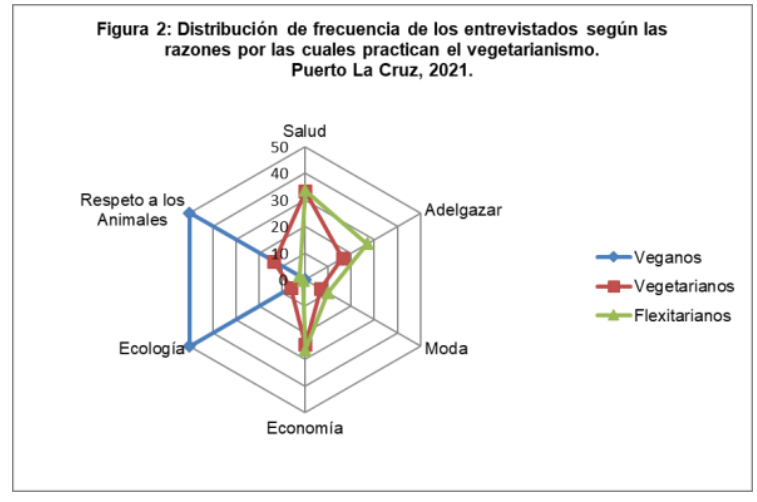

\section{Discusión}

Esta investigación muestra una panorámica alimentaria de un grupo de personas vegetarianas, como línea base para reconocer sus características y profundizar en el estudio de esta importante población. Este comportamiento alimentario logra ser adecuado para muchos de ellos, sin embargo, una buena parte requiere hacer cambios importantes y más o menos profundos en sus dietas para alcanzar criterios saludables.

No existen dudas relacionadas con los beneficios de las dietas vegetarianas y veganas (Rocha, Laster, Parag y Shah, 2019), la postura de la Asociación Americana de Dietética ha sido categórica al calificarlas como saludables y nutricionalmente adecuadas, capaces de proporcionar beneficios para la salud en la prevención y en el tratamiento de ciertas enfermedades. Destaca además que las dietas vegetarianas bien planificadas son apropiadas para todas las etapas del ciclo vital, incluidos el embarazo, la lactancia, la infancia, la niñez y la adolescencia, así como para deportistas; y que en algunos casos podrían resultar útiles los suplementos o los alimentos enriquecidos dadas sus grandes cantidades de nutrientes esenciales que logran proporcionar (Craig y Mangels, 2010). Sin embargo, a pesar de todo esto, parece existir un gran desconocimiento al respecto de estas opciones dietéticas tan importantes (Martínez, Ros y Nieto, 2019).

La evaluación antropométrica permitió evidenciar si bien la mayor parte de la muestra cursa con un diagnóstico normal $(73,21 \%)$, un $24,03 \%$ de personas evidencian sobrepeso $u$ obesidad, básicamente entre los grupos de vegetarianos y sobre todo de flexitarianos. De igual modo se evidenció la 


\section{Artículo Original}

presencia de $2,67 \%$ de déficit asociado exclusivamente a dietas vegetarianas y veganas. Parece existir una tendencia hacia un estado antropométrico-nutricional más saludable en la misma medida en que la dieta se configura sin carnes y con menos productos derivados de animales, lo cual incide de manera directa y evidente sobre la cantidad de energía que aporta la dieta.

La fórmula calórica de cada grupo corrobora la tendencia a disminuir la cantidad de grasas y proteínas consumidas, según se va haciendo más restrictiva la dieta con el consumo de alimentos de origen animal en general. En promedio los veganos consumen menos calorías que los restantes grupos estudiados, y dicha ingesta se sustenta más ampliamente en los carbohidratos de la dieta. Mientras que los grupos de vegetarianos y flexitarianos, al consumir productos de origen animal, estructuran una fórmula calórica con cuotas de participación de grasas más amplias, principalmente a expensas de los carbohidratos, y consecuentemente con una mayor carga calórica aportada. Esto corresponde con las referencias que observan, al igual que en nuestro estudio, variaciones en la prevalencia de obesidad entre vegetarianos y no vegetarianos; los primeros tienden a ser más delgados y presentar un peso inferior que los segundos. Algunos datos sugieren que la alimentación puede ser menos calórica mientras más estrictamente vegetariana se defina, que en cualquier caso es de menor densidad calórica (mucha fibra y menos grasa) y que define un metabolismo basal más elevado en los vegetarianos (Puiggròs Llop, 2008).

La fórmula calórica para cada grupo mostró esos cambios, definiéndose como adecuada únicamente la presentada por los vegetarianos ya que se ajustaba en todos sus macronutrientes a los valores de referencia establecidos para la población venezolana; la fórmula calórica vegana según este indicador se queda corta en la proporción de proteína suministrada $(8,9 \%)$, aunque otras referencias internacionales podrían avalar como adecuado este rango porcentual (Puiggròs Llop, 2008), mientras que la fórmula calórica evidenciada en los flexitarianos resultó desproporcionada para todos los macronutrientes.

Tanto la cantidad total de proteína consumida como la combinación de proteína vegetal para cubrir con los requerimientos necesarios de los aminoácidos esenciales, resulta un tema polémico para la población vegetariana; y a la fecha no existen consensos de organizaciones internacionales del área de la nutrición que emitan valores de referencia sobre la ingesta de proteínas de bajo valor biológico, corregidos justamente en función de la calidad proteica. Esto es particularmente crítico para el grupo de veganos, con los consumos porcentuales más bajos de proteínas y todas de origen vegetal, quienes en otros estudios venezolanos similares han demostrado desempeños controvertidos: población adulta con antropometría y bioquímica adecuada en unos, pero en otros se reportan niños y adolescentes con indicadores de composición corporal disminuidos (Guzmán Cáceres, 2016; Guzmán Cáceres, 2017).

Las cantidades de lípidos requeridas por los humanos perfectamente pueden ser cubiertas a través de cualquiera de las dietas vegetarianas propuestas, sin embargo, la discusión al respecto de la grasa gira alrededor de la calidad de las mismas. Las fuentes vegetales de algunos ácidos grasos poliinsaturados son limitadas, y solo una también limitada síntesis endógena podría suplir parte de su aporte dietético (Kaur, Chugh y Gupta, 2014; Tocher, Betancor, Sprague, Olsen y Napier, 2019). Este aporte se hace aún más crítico si la cantidad total de grasa ingerida tampoco es suficiente, como lo han demostrado particularmente parte del grupo de veganos estudiado en esta investigación.

Se están estudiando los beneficiosos efectos protectores cardiovasculares, neurológicos y cognitivos de los AGP (Sanders, 2014; Zarate, El Jaber-Vazdekis, Tejera, Pérez, y Rodríguez, 2017), particularmente de los ácidos grasos eicosapentaenoico (EPA) y docosahexaenoico (DHA), que son los más especialmente comprometidos en dietas sin alimentos de origen animal. Los vegetarianos no muestran signos clínicos de deficiencia de AGP, pero se requiere más investigación para determinar si los niveles observados en los vegetarianos son suficientes para mantener una salud óptima. Se sugiere a los vegetarianos tomar medidas dietéticas prácticas para optimizar la calidad de las grasas consumidas, como mejorar la ingesta de ácidos grasos omega-3, especialmente de ácido $\alpha$-linolénico (ALA) que es precursor de los EPA y DHA; así como procurar que 


\section{Artículo Original}

la grasa principal de la dieta proceda de alimentos y aceites ricos en oleico, como las aceitunas y el aceite de oliva, pero también otras grasas locales como anacardos, maní, y aguacates. Por otro lado, debería evitarse un exceso de alimentos ricos en ácido linoleico. Asimismo, la dieta no debería contener un exceso de ácidos grasos trans, procedentes de la hidrogenación de las grasas, que también interferiría en la deseada síntesis endógena de AGPI-CL n-3. (González Corbella, 2005; Saunders, Davis y Garg, 2013). La ingesta de ALA en vegetarianos también puede aumentarse mediante el consumo de semillas de linaza, semillas de chía o de micro algas (GarcíaMaldonado, Gallego-Narbón y Vaquero, 2019).

Los diferentes tipos de dieta vegetarianas estudiados, también evidencian una amplia capacidad de adecuación para el consumo de los micronutrientes analizados en esta investigación. En promedio todos los grupos demostraron ingestas mayoritariamente suficientes en cuanto a minerales ( $\mathrm{Ca}, \mathrm{Fe}, \mathrm{Mg}$ y $\mathrm{Zn}$ ) y vitaminas (A, C y B12), sin embargo sobre los que se discute actualmente a nivel internacional ya no solo se refiere a su cantidad adecuada sino también a su biodisponibilidad o calidad biológica (Pilis, et al., 2014; Rizzo, et al., 2016; Gluba-Brzózka, Franczyk y Rysz, 2017; Rojas Allende, et al., 2017; Torres Flores, Mata Ordoñez, Pavia Rubio,Rios Quirce y Sánchez Oliver, 2017; García Maldonado, et al., 2019)

En el caso del calcio la adecuación de consumo fue ampliamente alcanzada por vegetarianos y flexitarianos, también por la mayoría de veganos, aunque en un $13,79 \%$ de este último grupo se evidenció déficit del mineral. Esta adecuación baja potencia para este grupo el efecto negativo de otros factores propios de la naturaleza del calcio de origen vegetal en alimentos que limitan su absorción, como la habitual presencia concomitante de oxalatos, taninos y fitatos, así como la ausencia de lactosa, que no está presente en la dieta vegana y es un factor que favorece la absorción de calcio (Guzmán Cáceres, 2016). Considerando que los vegetales contienen cantidades apreciables de calcio, medidas de educación nutricional podrían ayudar a este grupo de la población estudiada a tomar decisiones dietéticas más asertivas, adicionalmente la adecuación del consumo de magnesio ha resultado excesiva para todos los grupos y esto podría ser una buena garantía pues el magnesio juega un papel importante en la absorción intestinal del calcio.

Muchas investigaciones (Rogerson,2017; Rose y Strombom, 2019; Bakaloudi, et al., 2020) destacan la necesidad de llevar a cabo una vigilancia en el consumo de zinc y hierro, especialmente de este último, puesto que el de origen vegetal se absorbe peor que el de origen animal y puede provocar problemas de anemia. El hierro proveniente de la dieta vegetariana es de naturaleza férrico (no-hem) el cual se une a los fitatos, taninos, fosfatos, oxalatos, fosfoproteínas, fibra, minerales (calcio, zinc, magnesio, manganeso y cobre) en los alimentos vegetales y éstos tienen un efecto inhibitorio en su absorción (Rojas Allende, et al., 2017) La población estudiada ha reportado una amplia adecuación en cuanto al hierro, pero en el caso del zinc ha evidenciado un déficit importante para el grupo de veganos.

Esta situación podría verse compensada ya que estas dietas vegetarianas han demostrado una amplia oferta de cantidades de vitaminas A y C, que son conocidos factores que favorecen la absorción y movilización de hierro no hemínico, además de disminuir el efecto inhibitorio que provocan los fitatos y polifenoles presentes en la dieta (Boccio, et al., 2003; Portillo, Fajardo, Solano y Barón, 2009; Butler y Ghugre, 2020). Sin embargo, contextualizando la adecuación del consumo de hierro en la población venezolana, resulta oportuno acotar que este mineral se encuentra en la lista de las deficiencias de micronutrientes y que la misma no es solo un riesgo para los vegetarianos, sino un problema de salud pública para Venezuela (Ekmeiro Salvador, Moreno Rojas, García Lorenzo y Cámara Martos, 2015; Guzmán Cáceres, 2016).

De igual modo y al respecto de la ingesta de zinc en la dieta de los vegetarianos, algunos autores han cuestionado su biodisponibilidad y señalado que, debido a su ineficiente absorción, incluso las ingestas excesivas, terminan siendo deficitarias (Foster y Samman, 2015). La Asociación Americana de Dietética sugiere que las necesidades de zinc para vegetarianos cuyas dietas son ricas en fitatos pudieran exceder las recomendaciones dietéticas (García Maldonado, et al., 2019); sin embargo, los efectos inhibidores del fitato sobre la absorción de zinc pueden minimizarse mediante métodos 


\section{Artículo Original}

modernos de procesamiento de alimentos como remojo, calentamiento, brotación, fermentación y uso de levaduras. Así la absorción de zinc puede mejorarse mediante el uso de panes a base de levadura y panes de masa madre, brotes y legumbres remojadas (Saunders, Craig y Baines, 2013).

El principal problema de las dietas vegetarianas es que son pobres en vitamina B12, como lo corroboran nuestros datos obtenidos donde se apreciaron deficiencias críticas para todos los grupos de participantes. La vitamina B12 se encuentra casi exclusivamente en alimentos de origen animal y, por lo tanto, es un nutriente de posible preocupación para quienes siguen una dieta vegetariana o vegana (Zeuschner, et al., 2012); incluso algunos investigadores explican que a pesar de la creencia de que el consumo de lácteos y huevos puede completar las necesidades de vitamina B12, es necesario siempre suplementar, tanto en vegetarianos como en veganos (Torres Flores, et al., 2017; Rudloff, et al., 2019).

Por tanto, la única suplementación que debería ser implementada por defecto en veganos, y cualquier persona que limite significativamente la ingesta de alimentos de origen animal, es la de vitamina B12 (Martínez, et al., 2019); sin embargo, evidenciamos en nuestro estudio que casi dos tercios de los encuestados lo ignoraban, y que la mitad de los participantes que si conocían la necesidad de la suplementación no podían, por diversas razones, cumplirla de forma regular.

Así, aunque tradicionalmente las investigaciones sobre vegetarianismo se han centrado en las posibles deficiencias nutricionales, en los últimos años el enfoque ha cambiado radicalmente y muchos más estudios se han orientado a confirmar los beneficios y ventajas para la salud de comer sin carne. Las personas se vuelven vegetarianas por muchas razones, que incluyen salud, convicciones religiosas, preocupaciones sobre el bienestar animal o el uso de antibióticos y hormonas en el ganado, o el deseo de comer de una manera que evite el uso excesivo de los recursos ambientales. Razones que está permitiendo al vegetarianismo transitar en la actualidad por uno de sus momentos evolutivos de mayor crecimiento y consolidación; determinando nuevos estilos de vida que poco a poco van transformando los sistemas culinarios (Contreras Hernández y García Arnaiz,
2005; Harvard Medical School, 2010; Rizzolo, 2018), e incidiendo progresivamente en las estructuras políticas y agroalimentarias tradicionales. Sin embargo, los niveles de compromiso y comensalidad no son iguales ni uniformes para todas las personas vegetarianas, que antes de ser definidas desde una esperada homogeneidad podríamos más bien englobarlas dentro de un "proceso" en que va alcanzando individualmente diferentes grados de pericia. Esta población es muy heterogénea, tanto como los son las razones por las cuales deciden modificar su conducta alimentaria, y por lo cual obliga a observarlos y estudiarlos como una población difusa alrededor de un creciente núcleo duro que sistematiza los fundamentos del grupo.

Esta diversidad evolutiva entre las personas participantes en esta investigación, ha quedado en evidencia al consultar las razones por las cuales se han incorporado a la cultura vegetariana. Flexitarianos y vegetarianos demostraron ser llevados por los mismos criterios, aunque en diferente grado de compromiso. Para los flexitarianos la motivación por la salud, pero particularmente por adelgazar, capitalizaba a la mayoría de los entrevistados; vinculada fuertemente por los referentes de la moda, muchos de ellos habían sido estimulados por los mediatizados ejemplos de artistas consagrados, modelos profesionales o deportistas exitosos que han hecho tendencia el vegetarianismo. En grupo de vegetarianos la motivación por la salud sigue siendo la más importante, pero incluyendo en una menor frecuencia el objetivo de adelgazar, lo que unifica el buen estado de salud como la más importante entre la totalidad de la muestra estudiada. Reconocen con esto la enorme incidencia de la dieta sobre el bienestar general, y sobre la eventual recuperación de estados patológicos, pero también los beneficios de las dietas vegetarianas sobre la salud los debemos analizar dentro de una perspectiva más amplia pues otras investigaciones han demostrado que los vegetarianos llevan estilos de vida más conscientes sobre la influencia de la actividad física, se abstienen de fumar y consumir alcohol con más frecuencia que lo no vegetarianos, además que han demostrado perfiles culturales más altos y frecuentemente pertenecen a niveles socioeconómicos más elevados (Rudloff, et al., 2019). 


\section{Artículo Original}

Otro elemento vinculante a propósito de la motivación entre flexitarianos y vegetarianos resulto el factor económico. Un porcentaje importante en ambos grupos de entrevistados destacaron que la adquisición mucho más limitada en cantidad y frecuencia de carnes y productos de origen animal, que tradicionalmente han sido de los alimentos más costosos en el mercado, incide positivamente en su presupuesto; lo cual propone seguir investigando sobre la vinculación del poder adquisitivo, el nivel socioeconómico, y el acceso real a los alimentos de esta población. El factor económico, junto al acceso a información nutricional realmente técnica, podrían eventualmente estar vinculado a la imposibilidad de incorporar regularmente alimentos que ayudarían a brindarle una mejor adecuación a sus dietas en algunos de los nutrientes estudiados; es el caso de semillas y frutos secos (anacardos, maní, ajonjolí, linaza, etc.) así como el aguacate, algas y algunos tipos de aceites (oliva, canola, semilla de uva) cuyos precios en el mercado podrían ser actualmente de difícil acceso para el consumidor promedio y por ello ver limitado el consumo de los mismos. Del mismo modo, el tema económico podría estar incidiendo en la imposibilidad de la suplementación regular con vitamina B12 que estas personas requieren regularmente.

Por otro lado, los veganos tienen una visión muy distinta y depurada de razones que justifican sus prácticas alimentarias. Señalan, a través de nuestro estudio, que contrarrestar los deterioros ecológicos en el planeta debido en gran medida a nuestros actuales patrones de consumo (incluyendo el alimentario) y el maltrato propiciado a los animales en la cadena agroalimentaria, son los criterios fundamentales de su veganismo. En menor grado en el grupo de vegetarianos algunos entrevistados ya proponían estas premisas, pero son los veganos quienes las hacen fundamentales; demostrando un probable trayecto evolutivo en la profundización y consolidación de hábitos alimentarios basados en productos vegetales más exclusivos, y con un carácter contestatario y alternativo hacia un modelo alimentario cuestionado en múltiples ámbitos por sus impactos negativos e indeseables tanto en lo personal como en lo colectivo.

Estas reflexiones podrían tener implicaciones para educar nutricionalmente; a los consumidores en general para a comer de manera más saludable basándose en productos naturales y de origen vegetal, así como en aquellos que ya han asumido patrones dietéticos vegetarianos para adaptar los contenidos de formación al momento evolutivo que transitan, haciendo de la educación nutricional una herramienta más útil y pragmática. De igual modo esta caracterización descrita, heterogénea pero inclusiva a la vez en las formas de "ser" vegetariano, posee implicaciones para productores y vendedores de alimentos, quienes tienen un enorme desafío para adaptar sus estrategias a las nuevas tendencias en alimentación y consumo (Ion 2007).

\section{Conclusiones}

En general la muestra evaluada y categorizada en veganos, vegetarianos y flexitarianos, evidenció una mayoritaria y amplia adecuación a través de los parámetros antropométricos y dietéticos estudiados. Sin embargo, más particularmente, solamente el grupo de vegetarianos logró un perfil calórico proporcionado dentro de los valores de referencia establecidos para la población venezolana.

Aunque los participantes demostraron ingestas adecuadas de grasas poliinsaturadas, se encontraron déficits marcados para los grupos de vegetarianos y veganos. Sobre el grupo de minerales estudiados se observó una amplia adecuación para el calcio, zinc, magnesio y hierro para el grupo general; pero se encontraron diferencias significativas de déficit de calcio y zinc en veganos, así como excesos de hierro y magnesio en los grupos de vegetarianos y veganos. Todas las categorías muestrales demostraron una amplia oferta de vitaminas A y C en sus dietas, así como también un notable déficit general de B12.

Aunque los criterios motivacionales por los cuales las personas entrevistadas se definían como vegetarianas fueron muy heterogéneos, se apreció una tendencia común entre flexitarianos y veganos por la salud y el adelgazamiento como razón principal; así como la economía que les puede representar una dieta libre o disminuida en productos de origen animal. Mientras que el grupo de veganos manifestó a la ecología y el maltrato animal como razones principales de su orientación alimentaria.

Estos resultados pueden tener implicaciones inmediatas e importantes en materia de educación nutricional; recomendando enfocar sus contenidos en 
función a las diferencias propias de cada categoría de la población vegetariana. De igual modo pueden ser la base para promocionar las ventajas nutricionales de un mayor y mejor consumo de alimentos de origen vegetal dirigidos a la población general, e importantes para productores y vendedores de alimentos ante la constante necesidad de adaptar sus estrategias a las nuevas tendencias en alimentación y consumo.

\section{Bibliografía}

Bakaloudi, D.R, Hallora, A., Rippin, H.L., Oikonomidou, A.C., Dardavesis, T.I., Williams, J., Wickramasinghe, K., Breda, J., y Chourdakis, M. (2020). Intake and adequacy of the vegan diet. A systematic review of the evidence. Clinical Nutrition, Article in Press. https://doi.org/10.1016/j.clnu.2020.11.035

Boccio, J., Salgueiro, J., Lysionek, A., Zubillaga, M., Goldman, C., y Weill, R. (2003). Metabolismo del hierro: conceptos actuales sobre un micronutriente esencial. Archivos Latinoamericanos de Nutrición, 53(2):119-132. http://ve.scielo.org/scielo.php?script=sci_arttext $\& \mathrm{pid}=$ S0004-

06222003000200002\&lng=es\&tlng=es.

Bravo, J.P., Ibarra, C.J., y Paredes M.M. (2014). Compromiso neurológico y hematológico por déficit de vitamina B12 en lactante hijo de madre vegetariana. Caso Clínico. Revista Chilena de Pediatría, 85(3),337-43. https://scielo.conicyt.cl/pdf/rcp/v85n3/art10.pdf

Bresnahan, M., Zhuang, J., y Zhu, X. (2016). Why is the vegan line in the dining hall always the shortest? Understanding vegan stigma. Stigma and Health, 1(1), 3-15. https://doi.org/10.1037/sah0000011

Brignardello, G.J., Heredia, P.L., Ocharán, S.M.P., y Durán, AS. (2013). Food knowledge of chilean vegetarians and vegans. Revista Chilena de Nutrición, $\quad 40(2), \quad 129-34$. http://dx.doi.org/10.4067/S071775182013000200006 .

Butler, N., y Ghugre, P. (2020). Effect of beta carotene on the ionisable iron content of wheat.
Current Research in Nutrition and Food Science, $8(1)$, 88-96. http://dx.doi.org/10.12944/CRNFSJ.8.1.08

Calderón Aravena, M.A. (2017). Estudio de prefactibilidad en la creación de una amasandería con productos de origen no animal. (Tesis de Grado). Universidad Andrés Bello, Santiago, Chile.

Centre de recherche pour l'étude et l'observation des conditions de vie (2019). Combien de végétariens en Europe? Synthèse des résultats à partir de l'étude "Panorama de la consommation végétarienne en Europe". https://www.franceagrimer.fr/Actualite/Filieres/ Viandes-rouges/2019/Conference-au-Sommetde-l-Elevage-evolution-de-la-consommation-deviande-et-panorama-du-vegetarisme-en-Europe

Chai, B.C., Van der Voort, J.R., Grofelnik, K., Eliasdottir, H.G., Klöss, I., y Pérez-Cueto, F.J.A. (2019). Which Diet Has the Least Environmental Impact on Our Planet? A Systematic Review of Vegan, Vegetarian and Omnivorous Diets. Sustainability, $\quad 11(15), \quad 4110$. https://doi.org/10.3390/su11154110

Clifford, J., y Kozil, A. (2017). Vegetarian diets. Colorado State University Extension. https://extension.colostate.edu/docs/pubs/foodnu t/09324.pdf

Cole, M., y Morgan, K. (2011). Vegaphobia: derogatory discourses of veganism and the reproduction of speciesism in UK national newspapers. The British Journal of Sociology, 62,134-53. https://doi.org/10.1111/j.14684446.2010.01348.x

Contreras Hernández, J., y García Arnaiz, M. (2005). Alimentación y cultura. Perspectivas antropológicas. Barcelona, España: Ariel.

Craig, W.J., y Mangels, A.R. (2010). Postura de la Asociación Americana de Dietética: dietas vegetarianas. Revista Española de Nutrición Humana y Dietética, 14 (1): 10-26. https://www.elsevier.es/es-revista-revistaespanola-nutricion-humana-dietetica-283- 
articulo-postura-asociacion-americana-dieteticadietas-X217312921049398X

Dinu, M., Abbate, R., Gensini, G.F., Casini, A., y Sofi, F. (2017). Vegetarian, vegan diets and multiple health outcomes: A systematic review with meta-analysis of observational studies. Critical Reviews in Food Science and Nutrition, 57(17), 3640-3649. https://doi.org/10.1080/10408398.2016.1138447

Ekmeiro Salvador, J., Moreno Rojas, R., García Lorenzo, M., y Cámara Martos, (2015). Patrón de consumo de alimentos a nivel familiar en zonas urbanas de Anzoátegui, Venezuela. Nutrición Hospitalaria, 32(4), 1758-1765. https://doi.org/10.3305/nh.2015.32.4.9404

Escuela de Nutrición y Dietética. (2002). Tabla de Raciones de Alimentos, Universidad Central de Venezuela. Facultad de Medicina. Caracas, Venezuela: UCV.

Foster, M., y Samman, S. (2015). Vegetarian diets across the lifecycle: impact on zinc intake and status. Advances in food and nutrition research, 74 , 93-131. https://doi.org/10.1016/bs.afnr.2014.11.003

García-Maldonado, E., Gallego-Narbón, A., y Vaquero, M.P. (2019). ¿Son las dietas vegetarianas nutricionalmente adecuadas? Una revisión de la evidencia científica. Nutrición Hospitalaria, 36(4), 950-961. https://dx.doi.org/10.20960/nh.02550

Gluba-Brzózka, A., Franczyk, B., y Rysz, J. (2017). Vegetarian diet in chronic kidney disease- A friend or foe. Nutrients, 9(4) ,374-389. https://doi.org/10.3390/nu9040374

González Corbella, M.J. (2005). Dietas vegetarianas. $\begin{array}{llll}O F F A R M, & 24 & \text { (5), } & \text { 82-90. }\end{array}$ https://www.elsevier.es/es-revista-offarm-4-pdf13074471

González Saura, V. (2018). Análisis de los efectos, beneficios y perjuicios de una dieta vegetariana en deportistas (Trabajo final de máster). Universitat de les Illes Balears, España.
Guzmán Cáceres, G.A. (2017). Alimentación, estilo de vida y su relación con la salud cardiovascular de los adventistas del 7 mo día del Distrito Capital. (Tesis de Maestría). Universidad Central de Venezuela, Caracas, Venezuela.

Guzmán Cáceres, R.G. (2016). Valoración del estado nutricional, de omega-3 y algunos micronutrientes en niños y adolescentes vegetarianos del Centro Occidente de Venezuela. (Trabajo Final de Especialización). Universidad Central de Venezuela, Caracas, Venezuela.

Hargreaves, S.M., Araújo, W.M.C., Nakano, E.Y., y Zandonadi, R.P. (2020). Brazilian vegetarians diet quality markers and comparison with the general population: A nationwide cross-sectional study. Plos One, 15(7): e0235991. https://doi.org/10.1371/journal.pone.0235991

Harvard Medical School. 2010. Becoming a vegetarian.

https://www.health.harvard.edu/stayinghealthy/becoming-a-vegetarian

Hölker, S., Von Meyer-Höfer, M., y Spiller, A. (2019). Animal Ethics and Eating Animals: Consumer Segmentation Based on DomainSpecific Values. Sustainability, 11(14), 3907. https://doi.org/10.3390/su11143907

Hrynowski, Z. (2019). What Percentage of Americans Are Vegetarian? https://news.gallup.com/poll/267074/percentageamericans-vegetarian.aspx

Instituto de Nutrición de Centro América y Panamá. (2012). Tabla de composición de alimentos de Centroamérica. Guatemala: INCAP/OPS.

Instituto Nacional de Nutrición. (2012). Valores de referencia de energía y nutrientes para la población venezolana. Caracas, Venezuela: Gente de maíz.

Instituto Nacional de Nutrición. (2015). Tabla de composición de los alimentos (Revisión 2012). Caracas, Venezuela: Gente de Maíz. 
Ion, R.A. (2007). Reasons why people turn to vegetanian diet. AgEcon Search, 54 (3), 353-358. https://ageconsearch.umn.edu/record/245701

Kahleova, H., Levin, S., y Barnard, N. (2017). Cardio-metabolic benefits of Plant-based diets. Nutrients, $9(8), \quad 848$. https://doi.org/10.3390/nu9080848

Kaur, N., Chugh, V., \& Gupta, A. K. (2014). Essential fatty acids as functional components of foods- a review. Journal of food science and technology, 51(10), 2289-2303. https://doi.org/10.1007/s13197-012-0677-0

Labbé Yáñez, D. (2016). El auge del veganismo en Chile. El Ciudadano. https://www.elciudadano.com/reportajeinvestigacion/el-auge-del-veganismo-enchile/01/09/

Le, L. T., Sabaté, J., Singh, P. N., y Jaceldo-Siegl, K. (2018). The Design, Development and Evaluation of the Vegetarian Lifestyle Index on Dietary Patterns among Vegetarians and NonVegetarians. Nutrients, 10(5), 542. https://doi.org/10.3390/nu10050542

Le Roy, C., Díaz San Martín, X. (2010). Dieta vegetariana en la edad pediátrica. Gastroenterología Latinoamericana, 21(1),9-14. https://gastrolat.org/DOI/PDF/10.0716/gastrolat 2010n100002.pdf

Martínez, A., Ros, G., Nieto, G. (2019). Estudio exploratorio del vegetarianismo en restauración colectiva. Nutrición Hospitalaria, 36(3), 681 690. http://dx.doi.org/10.20960/nh.2314

Martínez Biarge, M. (2019). Niños vegetarianos, ¿niños sanos? En: AEPap (ed.). Congreso de Actualización Pediatría 2019 (pp.65-77). Madrid, España: Lúa Ediciones.

Melina, V., Craig, W., y Levin, S. (2016). Position of the Academy of Nutrition and Dietetics: Vegetarian Diets. Journal of the Academy of Nutrition and Dietetics, 116 (12), 1970-1980. https://doi.org/10.1016/j.jand.2016.09.025
Menzel, J., Jabakhanji, A., Biemann, R., Mai, K., Abraham, K., y Weikert, C. (2020). Systematic review and meta-analysis of the associations of vegan and vegetarian diets with inflammatory biomarkers. Scientific Reports, 10, 21736. https://doi.org/10.1038/s41598-020-78426-8

Miranda, D.E., Gomes, A., Morais, J., Tonetti, T.A., y Vassimon, H. (2013). Qualidade nutricional de dietas e estado nutricional de vegetarianos. Demetra, $\quad 8(2), \quad 163-172$. https://doi.org/10.12957/demetra.2013.4773

Oregon State University. (2011). Why do people become vegans/vegetarians? Survey says: all of the above. https://today.oregonstate.edu/archives/2011/aug/ why-do-people-become-vegans-and-vegetarianssurvey-says-\%E2\%80\%93-all-above

Orellana, L.M., Sepúlveda, J.A., y Denegri, M. (2013). Significado psicológico de comer carne, vegetarianismo y alimentación saludable en estudiantes universitarios a partir de redes semánticas naturales. Revista mexicana de trastornos alimentarios, 4(1), 15-22. http://www.scielo.org.mx/scielo.php?script=sci_ arttext\&pid=S2007$15232013000100002 \& \operatorname{lng}=\mathrm{es} \& \ln \mathrm{ln}=\mathrm{es}$.

Parker, H.W., y Vadiveloo, M.K. (2019). Diet quality of vegetarian diets compared with nonvegetarian diets: A systematic review. Nutrition Reviews, $77(3)$, 144-60. https://doi.org/10.1093/nutrit/nuy067

Pilis, W., Stec, K., Zych, M., y Pilis, A. (2014). Health benefits and risk associated with adopting a vegetarian diet. Rocz Państwowego Zakładu Hig, 65(1), 9-14. https://pubmed.ncbi.nlm.nih.gov/24964573/

Portillo, Z., Fajardo, Z., Solano, L., y Barón, M.A. (2009). Consumo dietario de hierro y zinc, presencia de inhibidores y facilitadores de la absorción y conocimiento materno sobre el hierro como nutriente. Anales Venezolanos de $\begin{array}{llll}\text { Nutrición, } & 22 & \text { (2): } & \text { 76-83. }\end{array}$ https://www.analesdenutricion.org.ve/ediciones/ 2009/2/art-4/ 
Povey, R., Wellens, B., Conner, M. (2001) Attitudes towards following meat, vegetarian and vegan diets: an examination of the role of ambivalence. Appetite, 37(1) ,15-26. https://doi.org/10.1006/appe.2001.0406

Puiggròs Llop, C. (2008). Dieta Vegetariana. En: J. Salas-Salvadó (Ed.), Nutrición y dietética clínica (pp.465-477). Barcelona, España: Elsevier Masson.

Rizzo, G., Laganà, A.S., Rapisarda, A., Ferrera, G., Buscema, M., Rossetti, P., Nigro, A., Muscia, V., Valenti, G., Sapia, F., Sarpietro, G., Zigarelli, Micol., y Vitale, S.G. (2016). Vitamin B12 among Vegetarians: Status, Assessment and Supplementation. Nutrients, 8 (12), 767-790. https://doi.org/10.3390/nu8120767

Rizzolo, A. (2018). Rasgos y retos de la modernidad alimentaria - una entrevista con Jesús Contreras. Interface - Comunicação, Saúde, Educação, 22(67), 1267-1277. https://dx.doi.org/10.1590/1807-57622017.0383

Rocha, J.P., Laster, J., Parag, B., y Shah, N.U. (2019). Multiple Health Benefits and Minimal Risks Associated with Vegetarian Diets. Current Nutrition Reports 8, 374-381 (2019). https://doi.org/10.1007/s13668-019-00298-w

Rogerson, D. (2017). Vegan diets: practical advice for athletes and exercisers. Journal of the International Society of Sports Nutrition, 14, 36. https://doi.org/10.1186/s12970-017-0192-9

Rojas Allende, D., Figueras Díaz, F., y Durán Agüero, S. (2017). Ventajas y desventajas nutricionales de ser vegano o vegetariano. Revista Chilena de Nutrición, 44(3) ,218-225. http://dx.doi.org/10.4067/s0717-

75182017000300218

Rojas, Y.M. (2009). Evaluación dietética (Apéndice 2A). En: Henríquez G, Dini E, editores. Nutrición en Pediatría (pp.1269). Caracas, Venezuela: Centro de Atención Nutricional Infantil Antímano.

Rose, S.D., y Strombom, A.J. (2019). Ensuring Adequate Zinc Status in Vegans and Vegetarians.
Advanced Research in Gastroenterology \& Hepatology, $\quad$ 14(3): 555887. https://doi.org/10.19080/ARGH.2019.14.555887

Rosi, A., Mena, P., Pellegrini, N., Turroni, S., Neviani, E., Ferrocino, I., Di Cagno, R., Ruini, L., Ciati, R., Angelino, D., Maddock, J., Gobbetti, M., Brighenti, F., Del Rio, D., y Scazzina, F. (2017). Environmental impact of omnivorous, ovo-lacto-vegetarian, and vegan diet. Scientific Reports, $\quad 7(1), \quad 6105$ https://doi.org/10.1038/s41598-017-06466-8

Ruby, M. (2012). Vegetarianism. A blossoming field of study. Appetite, 58 (1), 141-150. https://doi.org/10.1016/j.appet.2011.09.019

Rudloff, S., Bührer, C., Jochum, F., Kauth, T., Kersting, M., Korner, A., Koletzko, B., Mihatsch, W., Prell, C., Reinehr, T., y Zimmer, K.P. (2019). Vegetarian diets in childhood and adolescence. Molecular and Cellular Pediatrics, 6, 4. https://doi.org/10.1186/s40348-019-0091-z

Sanders, T. A. (2014). Plant compared with marine n-3 fatty acid effects on cardiovascular risk factors and outcomes: what is the verdict? The American journal of clinical nutrition, 100 (Suppl 1), 453S-8S. https://doi.org/10.3945/ajcn.113.071555

Sánchez Mendoza, B., Flores Villalba, S., Rodríguez Hernández, E., Anaya Escalera, A.M., y Contreras Contreras, E.A. (2020). Causas y consecuencias del cambio climático en la producción pecuaria y salud animal. Revisión. Revista mexicana de ciencias pecuarias, 11(Supl. 2), 126145.https://doi.org/10.22319/rmcp.v11s2.4742

Saunders, A., Craig, W., y Baines, S. (2013). Zinc and vegetarian diets. The Medical journal of Australia, $\quad 199(4 \quad$ Suppl), $17-22$. doi.org/10.5694/mjao11.11493.

Saunders, A.B., Davis, B.C., y Garg M.L. (2013). Omega-3 polyunsaturated fatty acids and vegetarian diets. The Medical Journal of Australia, $\quad 199 \quad$ (S4), S22-S26. https://doi.org/10.5694/mja11.11507 
Sebastiani, G., Herranz Barbero, A., Borrás-Novell, C., Alsina Casanova, M., Aldecoa-Bilbao, V., Andreu-Fernández, V., Pascual Tutusaus, M., Ferrero Martínez, S., Gómez Roig, M.D., y García-Algar, O. (2019). The Effects of Vegetarian and Vegan Diet during Pregnancy on the Health of Mothers and Offspring. Nutrients, 11(3),557. https://doi.org/10.3390/nu11030557

The Nielsen Company. (2016). ¿Qué hay en nuestra comida y en nuestra mente? http://www.nielsen.com/content/dam/nielsenglo bal/latam/docs/reports/2016/Est udioGlobal_NuestraComidaYMente.pdf

Tocher, D. R., Betancor, M. B., Sprague, M., Olsen, R. E., y Napier, J. A. (2019). Omega-3 LongChain Polyunsaturated Fatty Acids, EPA and DHA: Bridging the Gap between Supply and Demand. $\quad$ Nutrients, $11(1), \quad 89$. https://doi.org/10.3390/nu11010089

Torres Flores, F., Mata Ordoñez, F., Pavia Rubio, E., Ríos Quirce, C., y Sánchez Oliver, A.J. (2017). Dieta vegetariana y rendimiento deportivo. Revista Digital de Educación Física, 8(46):2738. https://www.researchgate.net/publication/31575 2247_Dieta_vegetariana_y_rendimiento_ deportivo

United States Department of Agriculture. (2014). USDA Automated Multiple Pass Method. USDA. Agricultural Research Service. Features of AMPM.

http://www.ars.usda.gov/News/docs.htm?docid= 7710

Zarate, R., El Jaber-Vazdekis, N., Tejera, N., Pérez, J.A., y Rodríguez, C. (2017). Significance of long chain polyunsaturated fatty acids in human health. Clinical and Translational Medicine, 6(1):25. https://doi.org/10.1186/s40169-0170153-6

Zeuschner, C.L., Hokin, B.D., Marsh, K.A., Saunders, A.V., Reid, M.A., y Ramsay MR. (2012). Vitamin B12 and vegetarian diets. The Medical Journal of Australia; 199 (4), 27-32. 10.5694/mjao11.11509 